\title{
Gemeinsames Globales Lernen für den Klimaschutz. Die GLOBAL EDUCATION WEEK 2019 und das Studienbegleitprogramm STUBE Hessen
}

Die Schüler/-innen der Fridays-for-Future-Bewegung fordern Klimagerechtigkeit und sprechen damit ein Kernproblem der aktuellen Klimaschutzdebatte an: Viele Länder des Globalen Südens tragen nur in geringem Maße durch Emissionen zum Klimawandel bei, werden von dessen Folgen jedoch mit besonderer Härte getroffen. Das Globale Lernen will die Perspektiven der betroffenen Länder stärker in den Mittelpunkt rücken. Im Dialog über den weltweiten Klimaschutz ist es wichtig, dass allen Akteurinnen und Akteuren Gehör verschafft wird. Weltweite Partnerschaften müssen ernst genommen werden, wenn das Ziel der Klimagerechtigkeit erreicht werden soll. Zugleich betont Globales Lernen, dass der Klimawandel sich nicht nur auf ökologischer Ebene auswirkt. Genauso sind die soziale und wirtschaftliche Ebene betroffen. Das Zusammendenken dieser drei Aspekte - ökologisch, sozial und wirtschaftlich - ist auch für den Nachhaltigkeitsbegriff der UN-Agenda 2030 grundlegend.

Die diesjährige GLOBAL EDUCATION WEEK läuft unter dem Motto ,Wake up! Letzter Aufruf für den Klimaschutz'. Der Zusammenhang zwischen ökologischer, sozialer und wirtschaftlicher Nachhaltigkeit soll auch hier betont werden. Die Aktionswoche wird von dem europaweiten Global Education Network beim Nord-Süd-Zentrum des Europarates getragen und in Deutschland vom World University Service (WUS) koordiniert (www.globaleducationweek.de). Schulen, Universitäten, lokale Initiativen und Verbände warend im Aktionszeitraum vom 18. bis zum 24. November 2019 dazu eingeladen, sich mit eigenen Beiträgen zu beteiligen: mit Workshops, Videoclips, Ausstellungen und andere Aktivitäten, die zum Klimaschutz aufrufen und informieren.

Die GLOBAL EDUCATION WEEK bezieht sich auf die UN-Agenda 2030. Diese wurde im September 2015 von den Vereinten Nationen verabschiedet und folgt dem Motto „leave no one behind“. In siebzehn Nachhaltigkeitszielen (Sustainable Development Goals - SDGs ) werden Maßnahmen formuliert, deren Umsetzung bis 2030 erfolgen und allen Menschen weltweit ein würdiges Leben ermöglichen soll. Neben Themen wie der Bekämpfung von Armut und Hunger, Gesundheitsfürsorge und Bildung werden auch ökologische Themen behandelt: Maßnahmen für den Klimaschutz werden in Ziel 13 formuliert. Die Ziele 14 und 15 fordern einen achtsamen Umgang mit den Ressourcen und dem Leben im Wasser und an Land.

Neben den UN-Nachhaltigkeitszielen und der Komplexität des Nachhaltigkeitsbegriffes steht der Wissensaustausch zwischen Akteur/-innen aus Ländern des Globalen Südens und Nordens im Zentrum der Aktionswoche. Wie fruchtbar ein solcher Wissensaustausch sein kann, zeigt das WUS-Projekt STUBE Hessen - ein Studienbegleitprogramm für Studierende aus Afrika, Asien und Lateinamerika in Hessen (www.stube-hessen.de). Neben Seminaren und Akademien, die am Konzept der Bildung für nachhaltige Entwicklung (BNE) und den SDG ausgerichtet sind, bietet STUBE eine Förderung berufsvorbereitender Praktika- und Studienaufenthalte (bPSA) in den Herkunftsländern der Studierenden. Die STUBE Veranstaltungen und die Förderung von Aufenthalten in den Herkunftsländern verzahnen BNE: Das in Deutschland erarbeitete und reflektierte Wissen wird bei einem geförderten Aufenthalt im Globalen Süden angewandt. Die Teilnehmenden tragen somit zur Entwicklung des eigenen Landes bei und es wird eine nachhaltige Berufsperspektive für die Zeit nach dem Studium geschaffen.

Einer der Studierenden, den STUBE Hessen im Rahmen des bPSA-Programms unterstützt, ist Monir Hossen. Er studiert ,Economic Behaviour and Governance' an der Universität Kassel. Im Zuge seiner Masterarbeit forscht Hossen zu den volkswirtschaftlichen Kosten des Klimawandels für sein Herkunftsland Bangladesch. Seine Forschung beruht auf der zuvor dargelegten Problematik der Klimaungerechtigkeit: eine Situation, in der Länder des Globalen Südens die Folgen eines Problems tragen, das größtenteils von Ländern des Globalen Nordens verursacht wird. Zu seiner Forschung bewegte ihn die persönliche Erfahrung von Umweltverschmutzung, Flusserosion und Naturkatastrophen, wie beispielsweise Wirbelstürmen und Sturmfluten. Da die Landwirtschaft im ländlich geprägten Bangladesch nach wie vor von großer Bedeutung ist, haben diese Umstände verheerende Auswirkungen auf die Bevölkerung und die aufschwingende Wirtschaft des Landes. In seiner Masterarbeit evaluiert Hossen daher, inwieweit finanzielle Hilfen aus dem Ausland dabei unterstützen können, die Folgen der Erderwärmung abzufangen. Aktuell erhebt der Student dazu Daten vor Ort in Bangladesch.

STUBE Hessen-Teilnehmende profitieren vom inhaltlichen Austausch untereinander und bringen ihre Erfahrungen und Kenntnisse vielfach als Multiplikator/-innen in der entwicklungspolitischen Bildungsarbeit ein. Auch im Rahmen der GLOBAL EDUCATION WEEK stehen sie wieder gerne als Expert/-innen zur Verfügung.

Im Zuge beider Projekte wird ökologische, soziale und wirtschaftliche Nachhaltigkeit zusammengedacht und ein Dialog zwischen Akteur/-innen des Globalen Südens und Nordens ermöglicht. Hierbei zeigt sich, wie Bildung einen Beitrag zu mehr Klimagerechtigkeit leisten kann.

$$
\begin{array}{r}
\text { Katharina Schäfer, } \\
\text { Informationsstelle Bildungsauftrag Nord-Süd \& } \\
\text { Eileen Paßlack, } \\
\text { Studienbegleitprogramm (STUBE) Hessen für Studierende } \\
\text { aus Afrika, Asien und Lateinamerika; } \\
\text { World University Service - Deutsches Komitee e. V., } \\
\text { Informationsstelle Bildungsauftrag Nord-Süd, } \\
\text { www.informationsstelle-nord-sued.de } \\
\text { doi.org/10.31244/zep.2019.03.09 }
\end{array}
$$

